\title{
STANDING STABILITY EVALUATION USING A TRIAXIAL ACCELEROMETER
}

\author{
Ruth E. Mayagoitia, Joost C. Lötters, Peter H. Veltink \\ Biomedical Technology Institute, Department of Electrical Engineering, University of Twente, \\ P.O.Box 217, 7500 AE Enschede, The Netherlands \\ hill@servidor.unam.mx; J.C.Lotters@el.utwente.nl; P.H.Veltink@el.utwente.nl
}

\begin{abstract}
A triaxial accelerometer is placed at the back of the subject at the height of the center of mass. Force plate data are collected simultaneously. Subjects stand in a comfortable position with eyes open, eyes closed and doing cognitive tasks; and with feet together with eyes open and closed. The cognitive tasks are: mathematical, auditory Stroop and memory. The force plate data are processed to obtain the center of pressure and from it the parameters of: mean radius, speed and frequency, and base of support. The same parameters are obtained from the combined accelerations vector projection on the floor, found from the triaxial accelerometer data. The mean angular velocity, angular acceleration and accelerations in the horizontal plane at the level of the accelerometer are calculated. T-tests indicate that for most parameters the accelerometer measurements are able to distinguish between the different test conditions as well as the force plate $(p \leq 0.05)$.
\end{abstract}

\section{INTRODUCTION}

Many tests to evaluate postural stability have been developed [1]. These have concentrated more on the impairment aspect, depend on expensive equipment, can be difficult to interpret and are not standardized. A clinically useful test for evaluating a person's postural stability must focus on the amount of skill a person has to maintain the stability under different perceptual, cognitive, motor and mechanical conditions [2], be cheap to implement, easy to administer, and simple to interpret. Thus, the project objective was to develop a system for clinical application to perform standing clinical tests substituting the habitually used force platform with a triaxial accelerometer.

\section{MATERIALS AND METHODS}

A triaxial accelerometer developed at the University of Twente [3], mounted on a motorcyclist belt, is placed in the center of the back of the subject, at the approximatc height of the center of mass, while standing with the arms hanging by the side. For validation purposes, data have been collected at the same time from an AMTI force plate and from the triaxial accelerometer at $50 \mathrm{~Hz}$. There are seven test conditions, each lasting 30 seconds: feet in a comfortable position with eyes open, eyes closed and doing three cognitive tasks; and with . feet together with eyes open and closed. The cognitive tasks are: mathematical, auditory Stroop and memory, based on the ideas presented in [2] where a dual task should tax the balance control system and aid in distinguishing between apparently stable and truly stable individuals. All conditions are randomized and repeated twice.

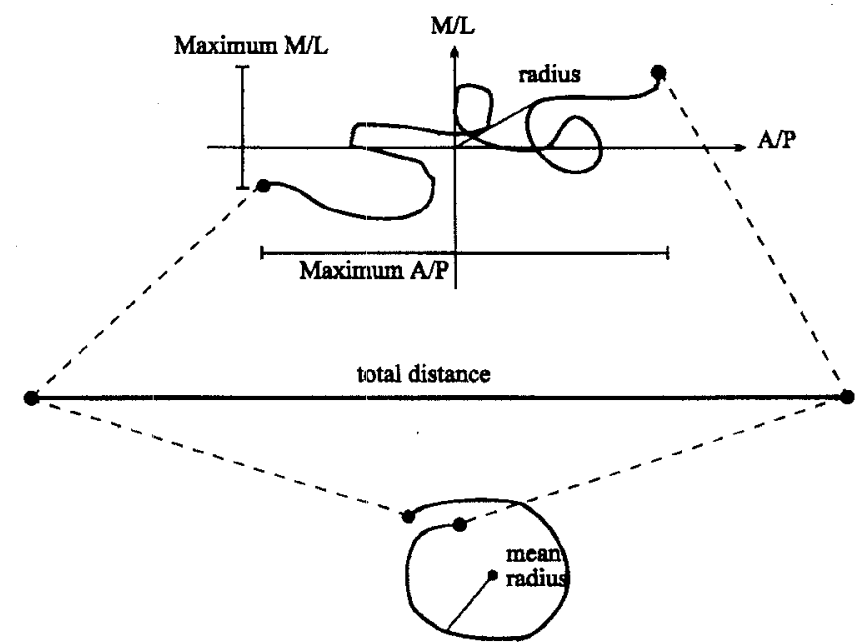

Figure 1. Sway path and parameter calculation for standing stability evaluation

The data are analyzed using a purpose written program to obtain several performance parameters based on [4]. Figure 1 contains a schematic representation of the way the parameters were found. The five parameters obtained from the force plate data are: mean speed, mean radius, mean frequency, and the maximum displacements in the anterior-posterior and medial-lateral directions.

The mean speed can be found using:

$\mathrm{V}=\Sigma \sqrt{ }\left(\left(\mathrm{d}_{\mathrm{x}}-\mathrm{d}_{\mathrm{x}-1}\right)^{2}+\left(\mathrm{d}_{\mathrm{y}}-\mathrm{d}_{\mathrm{y}-1}\right)^{2}\right) / \Delta \mathrm{t}$

Where $V$ is the mean speed, $d_{x}$ and $d_{y}$ are the coordinates at floor level, and $\Delta t$ is the time increment given by the sampling frequency. The mean radius is given by:

$R=\Sigma \sqrt{ }\left(\left(d_{x}-d_{x m}\right)^{2}+\left(d_{y}-d_{y m}\right)^{2}\right) / n$

Where $R$ is the mean radius, $d_{x m}$ and $d_{y m}$ are the mean of all $x$ or $y$ coordinates and $\mathrm{n}$ is the number of samples.

The mean frequency, $\mathrm{F}$, in $\mathrm{Hz}$ is given by:

$\mathrm{F}=\mathrm{V} / 2 \pi \mathrm{R}$ 
The mean radius is interpreted as inversely proportional to the stability. A low sway speed is associated with greater stability since it indicates smaller, better controlled adjusting movements are being made. If the mean frequency is of a similar value under different (destabilizing) conditions, such as closing the eyes, it is assumed that the person is able to compensate for these adverse conditions. The maximum displacements indicate the limits of the sway area.

A tracing of the travel of the end of the vector combining all three accelerations projected on the floor can be obtained during quiet standing, using the data from the triaxial accelerometer, according to figure 2 . The same performance parameters can then be calculated.

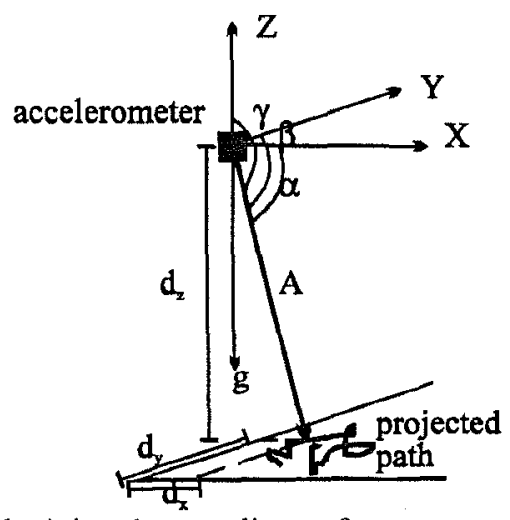

Figure 2. Obtaining the coordinates for a sway path at ground level from triaxial accelerometer data.

\section{RESULTS}

T tests were done to compare the results obtained by the different measurement systems and under the different test conditions. Table 1 contains some of the statistical significances of the results of tests done with eight young healthy male volunteers. Thus, the parameters found with the triaxial accelerometer are able to distinguish between test conditions as well as the results from the force plate.

Also, the three sets of dual task results were compared to comfortable position eyes open results and in none of the cases for either of the measurement systems could the null hypothesis be rejected, except for the mean frequency, where it could be rejected in all of the cases. All these results are what would be expected of normal individuals.

\section{DISCUSSION}

Further tests using a new, more sensitive accelerometer are being carried out and it is hoped that its use will further allow the performance parameters obtained from the accelerometer to behave in the same way as the force plate derived results, with the results having the same sort of clinical interpretation.

The parameters of angular (alpha) and plane (mean xy) acceleration derived by direct application of the inverted pendulum model need to be further studied in order to reach statistically applicable results, but they appear to be useful performance indicators in addition to those described in detail above.

The triaxial accelerometer based system is: very easy to use, unobtrusive to movement of the subject and cheap. By collecting the data on a portable data logger, it can be used in any setting. The results are easy to interpret. Dual task tests can help to discern between subjects who are apparently stable and those who are truly stable. A test based on the accelerometer is being implemented for routine clinical use.

This system is suitable for other applications such as for control of functional electrical stimulation standing systems.

Table 1. Statistical Significances

*= null hypothesis can be rejected at $\mathrm{P}<0.05$ level

feet comfortable position, feet together, eyes eyes open vs. eyes closed open vs. eyes closed

\section{Force Plate}

mean speed

mean radius

mean freq

maximum $x$

maximum y

Accelerometer

mean speed

mean radius

mean freq

maximum $x$

maximum $y$

alpha

mean xy accel

$\begin{array}{ll}* & * \\ 0.20 & * \\ * & * \\ * & * \\ * & * \\ * & \\ * & * \\ 0.15 & * \\ * & * \\ 0.41 & * \\ * & * \\ * & * \\ & *\end{array}$

\section{REFERENCES}

[1] R E Mayagoitia: Stability in FES and Orthosis Assisted Paraplegic Standing. Glasgow, Scotland: University of Strathclyde, PhD Thesis, 1989.

[2] A C H Geurts, T W Mulder, B Nienhuis, R A J Rijken: Dual-task assessment of reorganization of postural control in persons with lower limb amputation. Archives of Phys Med and Rehab, vol 72, pp 1059-1064, 1991.

[3] J C Lötters, W Olthuis, P H Veltink, P Bergveld: On the design of a triaxial accelerometer. J. Micromech Microeng, vol 5, pp 128-131, 1995 .

[4] G R Cybulski, R J Jaeger: Standing performance of persons with paraplegia. Archives of Phys Med and Rehab, vol 67, pp 103-108, 1986. 